\title{
Modeling of high-index coating lensed fibers for silicon nanophotonic device coupling
}

\author{
Jeong Hwan Song \\ jeonghwan.song@tyndall.ie \\ Ko-Hsin Lee
}

Frank H. Peters

\author{
Integrated Photonics Group, Tyndall National Institute, Lee Maltings, Cork, Ireland \\ Integrated Photonics Group, Tyndall National Institute, Lee Maltings, Cork, Ireland \\ Integrated Photonics Group, Tyndall National Institute, Lee Maltings, Cork, Ireland \\ Physics Department, University College Cork, Cork, Ireland
}

Two types of high-index coated lensed fibers are numerically investigated for optical coupling from Si-based nanowire waveguides to the lensed fibers. One is with a layer of high-index film coated on tip of conventional lensed single-mode fiber (SMF) and the other is with an additional coreless fiber section inserted in between. The simulation results show that, for nanowires with mode size diameters ranging from $0.6 \mu \mathrm{m}$ to $1.3 \mu \mathrm{m}$, the coupling efficiency as high as $80 \%$ can be obtained with the former type of fiber when the radius of curvature is around $10 \mu \mathrm{m}$ and coated with a 5-10 $\mu \mathrm{m}$ thick high-index film. As for the latter design of fiber, an improved working distance is calculated to be as long as $36.8 \mu \mathrm{m}$ by inserting a coreless fiber section. Both high-index coating lensed SMF designs show potential application for coupling with Si-based nanophotonics. [DOI: 10.2971/jeos.2010.10007]

Keywords: fiber lens, nanowire coupling, Gaussian approximation, fiber device, optical fiber

\section{INTRODUCTION}

Silicon-based nanophotonic waveguides may enable the realization of high-density photonic integrated circuits due to unique properties of the extremely high refractive index contrast. Designs like sharp waveguide bends with radii of only a few microns have been reported on silicon nanowires based on silicon-on-insulator (SOI) [1]. However, such a high index contrast can result in a large mode size mismatch between the fundamental mode of the Si nanowire waveguides and that of the single mode fiber (SMF), which are a few hundred nanometers and ten micrometers, respectively; it then leads to a high coupling loss. Hence, the fiber coupling for Si-based photonic nanowire waveguides is a key technology to enable practical nano-Si photonics applications. Up to date, researchers have proposed several designs to enhance the coupling, such as grating couplers [1]-[3], spot size converters (SSCs) [4], and polymer-tipped optical fibers [5]. In addition, Shiraishi et al. [6] have recently reported a new scheme to reduce the spot size of a fiber by coating the highindex hydrated amorphous silicon $(a-\mathrm{Si}: \mathrm{H})$ material on the tip of conventional lensed SMF, which was theoretically analyzed by employing the two-dimensional finite-difference time-domain (2D-FDTD) calculation method and experimentally evaluated. Usage of a high-index coating has shown to enhance the focusing effect and to increase the numerical aperture (NA) of the lensed SMF up to $\approx 0.95$, so that a higher coupling efficiency is believed to be obtained for fiber coupling with nanowire waveguides.

Even though the FDTD method may give better accuracy for coupling performance calculations, the large demands of computation capacity and time are often undesirable. In this pa- per, we present a different numerical analysis method using Gaussian approximation in which the mode profile variations through media like the high-index coating layer and coreless fiber section are calculated via ABCD matrix [7]-[11]. To approximate the circumstance of lensed fiber with coating layers, a two-lens system is adopted in our analysis. Based on this concept, we establish and deduce the mathematical equations in Section 2 to model the case of coupling from Si-based nanowire waveguides with very small optical mode sizes $(<1 \mu \mathrm{m})$ to high-index coating lensed SMF. The simulated results can provide a guideline for fiber coupling with $\mathrm{Si}$ nanophotonic devices. In Section 3, we further apply our analysis method to investigate a novel structure of a high-index coating lensed SMF with a coreless fiber section for improved coupling performances. Finally, the conclusion of this work is presented in Section 4.

\section{HIGH INDEX COATING LENSED FIBER}

\subsection{Numerical analysis}

Our coupling design of Si-nanowire waveguide to high-index coated lensed SMF and the notations of physical parameters are shown schematically in Figure 1. The mode sizes of the waveguide and SMF are designated as $\omega_{0 w(x, y)}$ and $\omega_{0 f}$, respectively. The high-index coating material is characterized by the film thickness, $T_{\mathcal{c}}$, and the refractive index, $n_{c}$, which is larger than the refractive index of the fiber core $\left(n_{\text {core }}=1.45\right)$ and that of air $\left(n_{\text {air }}=1\right)$. Considering an ideal optical propagation with Gaussian profile for the fundamental mode emitting from the waveguide facet, its radius of curvature of the 


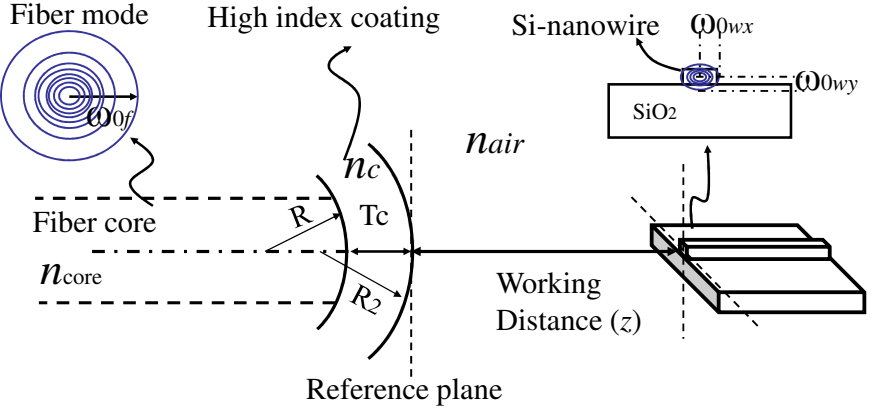

Lensed fiber tip
Si-nanowire device

FIG. 1 Schematics of coupling between Si-nanowire waveguide and high-index coating lensed single mode fiber and the relative notations.

wavefront, $r_{w}$, and the mode size, $\omega_{w}$, along the propagation distance can be expressed by

$$
\begin{gathered}
r_{w(x, y)}=z\left[1+\left(\frac{k \omega_{0 w(x, y)}^{2}}{2 z}\right)^{2}\right] \\
\omega_{w(x, y)}=\omega_{0 w(x y)} \sqrt{1+\left(\frac{2 z}{k \omega_{0 w(x, y)}^{2}}\right)^{2}}
\end{gathered}
$$

where $k=2 \pi / \lambda$ denotes the wave number, $\lambda$ is the wavelength in free space, and $z$ is the propagation distance from the waveguide facet and is also designated as the working distance when evaluating the coupling efficiency.

In general, the coupling efficiency between two optical fields $E_{1}(x, y)$ and $E_{2}(x, y)$ is defined as

$$
\eta=\frac{\left|\iint E_{1}(x, y) \cdot \overline{E_{2}(x, y)} d x d y\right|^{2}}{\left(\iint\left|E_{1}(x, y)\right|^{2} d x d y\right) \cdot\left(\iint\left|E_{2}(x, y)\right|^{2} d x d y\right)} .
$$

Here, we choose the interface at the tip of coating lensed fiber (in contact with air) as the reference plane to calculate the mode overlap integral. The optical coupling efficiency is then defined as the overlap integral of the optical field at this reference plane for light with fundamental mode coming from the waveguide, $\psi_{w}$ and the fiber optical field at the end of coating lens $\psi_{f}$. The subscripts $w$ and $f$ represent the waveguide and fiber, respectively. Therefore, Eq. (3) can be rewritten as

$$
\eta=\frac{\left|\iint \psi_{w}(x, y) \cdot Q \psi_{f}(x, y) d x d y\right|^{2}}{\left(\iint\left|\psi_{w}(x, y)\right|^{2} d x d y\right) \cdot\left(\iint\left|\psi_{f}(x, y)\right|^{2} d x d y\right)}
$$

where $Q$ is the transfer function of the focusing lens and is related to the focal length, $f$, given by

$$
Q=\exp \left[i k \frac{x^{2}+y^{2}}{2 f}\right] \text {. }
$$

In a system with radial coordinate, the $x$ and $y$ can be replaced by $\rho=\sqrt{x^{2}+y^{2}}$. Therefore, we can rewrite $\psi_{w}$ and $\psi_{f}$ approximately as $\psi_{w}=\exp \left(-\rho^{2} / \omega_{w}^{2}\right)$ and $\psi_{f}=\exp \left(-\rho^{2} / \omega_{f}^{2}\right)$, where $\omega_{w}$ and $\omega_{f}$ represent the fundamental mode sizes at the reference plane for lights coming from fiber and nanowire

waveguide, respectively. Moreover, by introducing a phase factor term [9], $\exp (2 i \pi L / \lambda)$, Eq. (4) can be rewritten as

$$
\eta=\frac{\left|\int_{0}^{\infty} \psi_{w} \psi_{f} t \exp \left[i \frac{2 \pi}{\lambda} L\right] \rho d \rho\right|^{2}}{\int_{0}^{\infty} \psi_{w}^{2} \rho d \rho \int_{0}^{\infty} \psi_{f}^{2} \rho d \rho}
$$

where $t$ is the transmission coefficient and $L$ is the parameter representing the phase error. It should be noticed that the actual mode from a nanowire waveguide is not symmetric $\left(\omega_{0 w x} \neq \omega_{0 w y}\right)$, so the coupling efficiency can be calculated with $\eta=\eta_{x} \eta_{y}$.

Furthermore, the optical propagation from a lensed SMF through a coating layer can be approached as a two-lens system. With implementation of the thick-lens approximation [7], the phase error $(L)$ and the corresponding characteristic $\mathrm{ABCD}$ matrix are expressed as

$$
\begin{aligned}
& L \cong \frac{n_{\text {air }}}{2}\left(\frac{1}{r_{w}}-\frac{1}{r_{f}}\right) \rho^{2} \\
&\left(\begin{array}{cc}
A & B \\
C & D
\end{array}\right)=\left(\begin{array}{cc}
1 & 0 \\
-\frac{1}{f_{\text {coating }}} & 1
\end{array}\right)\left(\begin{array}{cc}
1 & \frac{T_{c}}{n_{c}} \\
0 & 1
\end{array}\right) \\
& \times\left(\begin{array}{cc}
1 & 0 \\
-\frac{1}{f_{f}} & 1
\end{array}\right)\left(\begin{array}{cc}
1 & \frac{T_{f}}{n_{\text {core }}} \\
0 & 1
\end{array}\right)
\end{aligned}
$$

where $f_{\text {coating }}$ and $f_{f}$ are the focal lengths of curved coating layer and lensed SMF, respectively, and are expressed as $f_{\text {coating }}=R_{2} /\left(n_{c}-1\right)$ and $f_{f}=R n_{c} /\left(n_{\text {core }}-n_{c}\right)$. The $R_{2}$ and $R$ are radii of the coating layer and the lensed tip, respectively. $T_{\mathcal{C}}$ and $T_{f}$ represent the thickness of the coating layer and the lensed tip of the fiber; in which, we assume $T_{f}=0$ for simplicity. For the case of a cleaved-facet fiber, the focal length and the lens axial radius should be infinite. In our modelled coupling system, the focal length of two combined lens, $f$, and the fiber mode size at reference plane (tip of coating lensed fiber) can be written as

$$
\begin{gathered}
f=-\frac{1}{C} \\
\omega_{f}=A \omega_{0 f} \sqrt{1+\left(\frac{\lambda B}{A \pi \omega_{0 f}^{2}}\right)^{2}} .
\end{gathered}
$$

In a real lens system, the phase error term always exists. However, an lens system with shorter focal length, such as this type of lensed SMF coated with a high-index layer, the phase error can be minimized or nearly zero when $r_{f}=f$ [9]. Later in Section 3, we will show that, the coupling efficiency varies more visibly with phase error at a longer focal length. Therefore, the coupling efficiency is found to be maximum here by minimizing the phase error. Additionally, we assume that the transmission coefficient, $t$, goes to unity because the reflection coefficient can be as low as $0.5 \%$ with the help of sandwiched antireflection coatings on the high index layer [6] for practical applications. As for the propagation loss within coating layer section, it is estimated to be less than $0.2 \mathrm{~dB}$, which is a typical value for thin film filters with thicknesses of $25 \mu \mathrm{m}$ [12]. As the thickness distribution of the coating layer is not perfectly uniform, we assume that $R_{2}=R+T_{c} \times 25 \%$ [6]. 


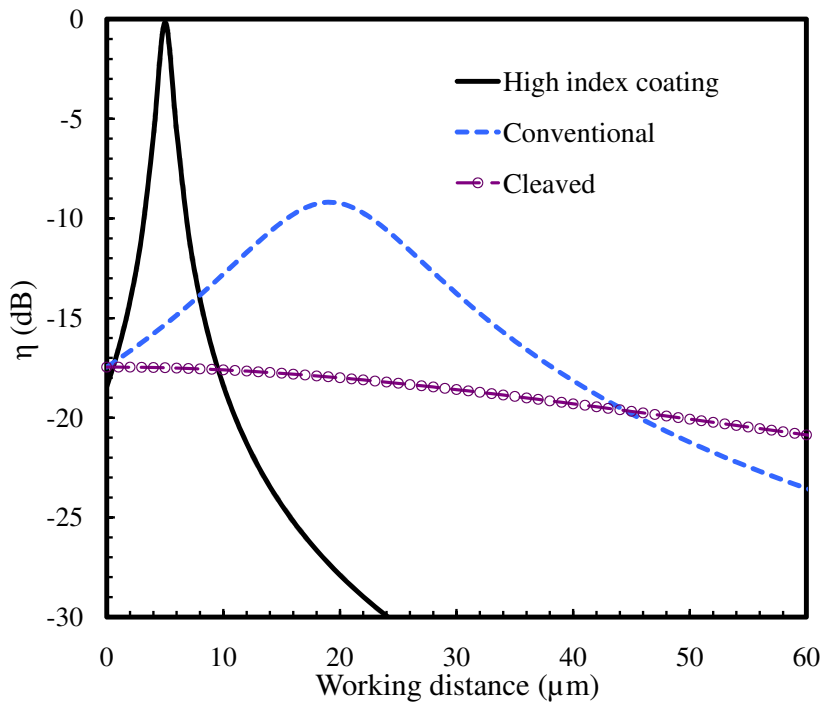

FIG. 2 Variation of coupling loss with respect to working distance for coupling between nanowire waveguide $\left(2 \omega_{0 w x}=2 \omega_{0 w y}=0.70 \mu \mathrm{m}\right)$ and different types of single-mode fibers $\left(2 \omega_{0 f}=10.4 \mu \mathrm{m}\right)$. The investigated fibers include the high-index coating lensed single mode fiber $\left(R=10 \mu \mathrm{m} ; T_{c}=10 \mu \mathrm{m}\right)$, the conventional lensed single mode fiber $(R=10 \mu \mathrm{m})$, and the cleaved single mode fiber $(R=\infty)$.

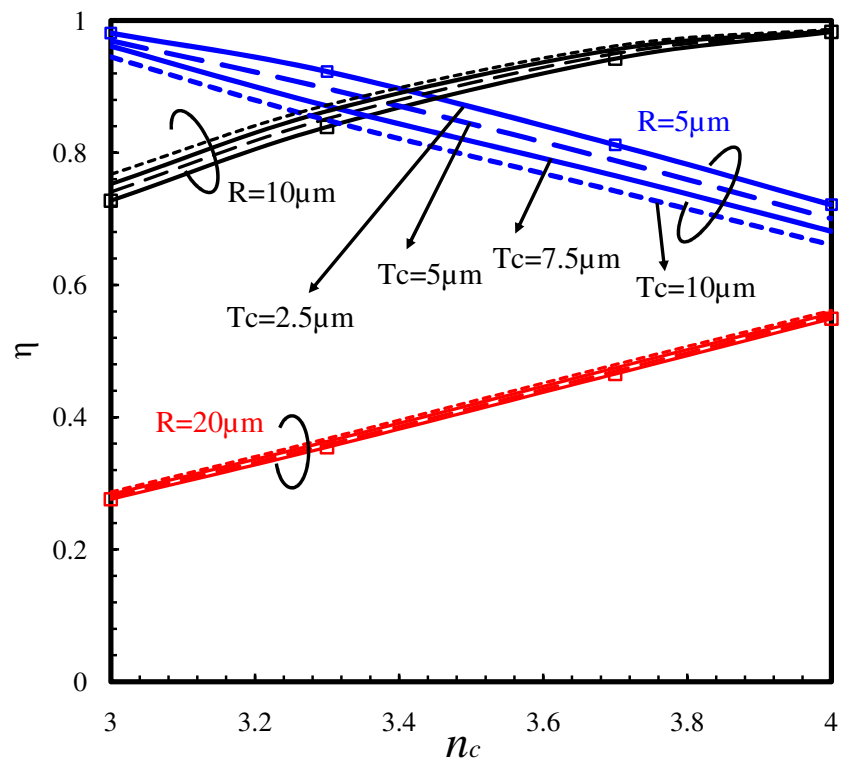

FIG. 3 Coupling efficiency of nanowire waveguide $\left(2 \omega_{0 w x}=2 \omega_{0 w y}=0.70 \mu \mathrm{m}\right)$ to the coating lensed single mode fibers with various coating layer index $\left(n_{c}\right)$, coating layer thicknesses $\left(T_{c}\right)$ and radius of curvature of tip lens $(R)$.

\subsection{Simulation results and discussion}

First, we evaluate the coupling efficiency, working distance, and alignment tolerance for designing the high-index coating lensed SMF when considering fiber coupling with nanowire waveguides. In the following simulations, the mode field diameters (MFD $=2 \times \omega_{0 w}$ ) of nanowire waveguide are set to $2 \omega_{0 w x}=2 \omega_{0 w y}=0.70 \mu \mathrm{m}$ and the MFD of SMF $\left(2 \omega_{0 f}\right)$ is $10.4 \mu \mathrm{m}$, corresponding to an acceptance angle, $\theta$, of $\pm 5.4^{\circ}$ using $\omega_{0(w, f)}=\lambda /\left(\pi \theta_{(w, f)}\right)[8]$ when $\lambda=1.55 \mu \mathrm{m}$. The investigated range for the radius of curvature of the lenses is between $5 \mu \mathrm{m}$ to $20 \mu \mathrm{m}$. The refractive index and thickness of the coating material vary from 3 to 4 , and $2.5 \mu \mathrm{m}$ to $10 \mu \mathrm{m}$, respectively.

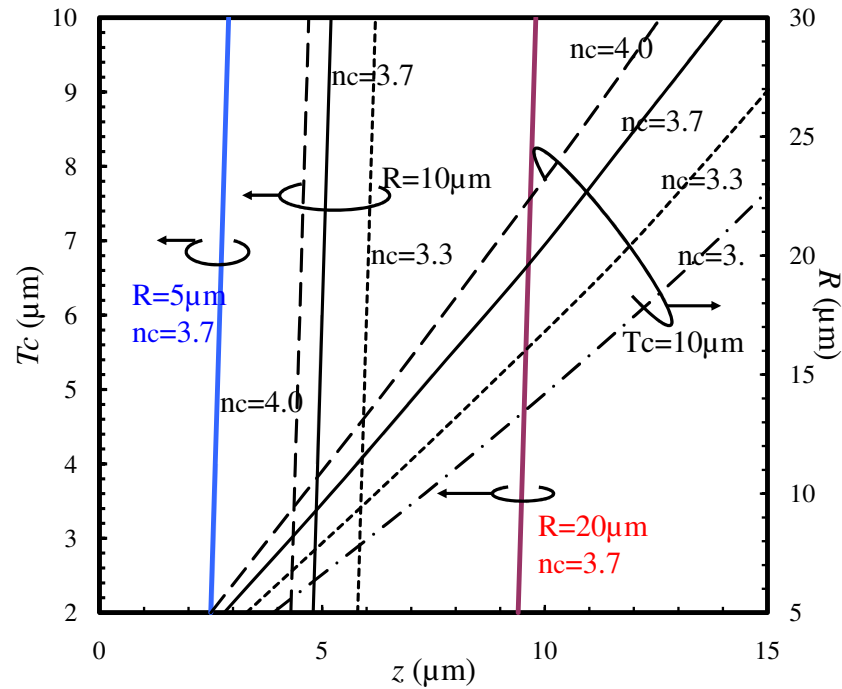

FIG. 4 Variation of working distance $(z)$ with coating layer thickness $\left(T_{c}\right)$, curvature radius $(R)$ for several different coating indices $\left(n_{c}\right)$ of lensed single mode fibers coupled to nanowire waveguide $\left(2 \omega_{0 w x}=2 \omega_{0 w y}=0.70 \mu \mathrm{m}\right)$.

Figure 2 shows the calculated coupling efficiency of nanowire waveguide to high-index coating lensed SMF, conventional lensed SMF, and cleaved SMF as a function of the working distance using our modeling method. The conventional lensed SMF is known to be more suitable for coupling compared to cleaved SMF because of a lower coupling loss; however, its coupling efficiency is often limited to less than $10 \%$ ( $R=10 \mu \mathrm{m}$ ) even at the optimized working distance. Using the high-index intermediate layer, the focusing effect can be highly enhanced. Hence the coupling efficiency increases and is found to be as high as $80 \%$ when using the coating lensed $\operatorname{SMF}\left(T_{c}=10 \mu \mathrm{m}, n_{c}=3.7\right.$, and $\left.R=10 \mu \mathrm{m}\right)$ at a $5.2 \mu \mathrm{m}$ working distance.

For further study, we analyze the relations among various coating layer thickness, $T_{c}$, coating layer index, $n_{c}$, and radius of curvature of tip lens, $R$, as shown in Figure 3. For $R=10 \mu \mathrm{m}$ and $20 \mu \mathrm{m}$, the coupling efficiency increases approximately linearly with $n_{c}$. Unlike the case of $R=5 \mu \mathrm{m}$, the coupling efficiency decreases when $n_{c}$ varies from 3 to 4 and a thinner coating layer is favored as well to obtain a higher coupling efficiency.

During the aligning and assembly process, the working distance must be considered for the design of lensed fibers since the facet of a nanowire waveguide structure could be damaged if the working distance is too short. To avoid damage of the nanowire waveguide facet, the working distance should be a few micrometers at a minimum. Figure 4 shows the working distance as a function of the coating layer thickness, index, and the radius of curvature of tip lens. From the figure, the variation of working distance is not sensitive to the change with coating layer thickness. Generally, the working distance increases with decreasing the coating layer index and increasing the radius of curvature of tip lens. Within our investigation range with coupling efficiency higher than $80 \%$ (see Figure 3) for $R=5 \mu \mathrm{m}$ and $10 \mu \mathrm{m}$, the working distance is about $2.8 \mu \mathrm{m}$ to $6.2 \mu \mathrm{m}$. The simulation results are summarized in Table 1 . 


\begin{tabular}{|c|c|c|c|c|c|c|c|c|}
\hline Conditions: & \multicolumn{2}{|c|}{$T_{c}=10 \mu \mathrm{m}$} & \multicolumn{2}{c|}{$T_{c}=10 \mu \mathrm{m}$} & \multicolumn{2}{c|}{$T_{c}=5 \mu \mathrm{m}$} & \multicolumn{2}{c|}{$T_{c}=5 \mu \mathrm{m}$} \\
$0.7 \times 0.7 \mu \mathrm{m}^{2}$ & \multicolumn{2}{c|}{$R=10 \mu \mathrm{m}$} & \multicolumn{2}{c|}{$R=5 \mu \mathrm{m}$} & \multicolumn{2}{c|}{$R=10 \mu \mathrm{m}$} & \multicolumn{2}{c|}{$R=5 \mu \mathrm{m}$} \\
\hline Coating layer index & $\eta$ & $\mathrm{z}(\mu \mathrm{m})$ & $\eta$ & $\mathrm{z}(\mu \mathrm{m})$ & $\eta$ & $\mathrm{z}(\mu \mathrm{m})$ & $\eta$ & $\mathrm{z}(\mu \mathrm{m})$ \\
\hline 3.0 & 0.76 & 7.3 & 0.94 & 3.9 & 0.74 & 7.0 & 0.97 & 3.7 \\
\hline 3.3 & 0.87 & 6.2 & 0.86 & 3.3 & 0.85 & 6.0 & 0.90 & 3.1 \\
\hline 3.7 & 0.92 & 5.2 & 0.74 & 2.8 & 0.95 & 5.0 & 0.79 & 2.6 \\
\hline 4.0 & 0.99 & 4.6 & 0.66 & 2.5 & 0.98 & 4.4 & 0.70 & 2.3 \\
\hline
\end{tabular}

TABLE 1 Summary of simulation results.

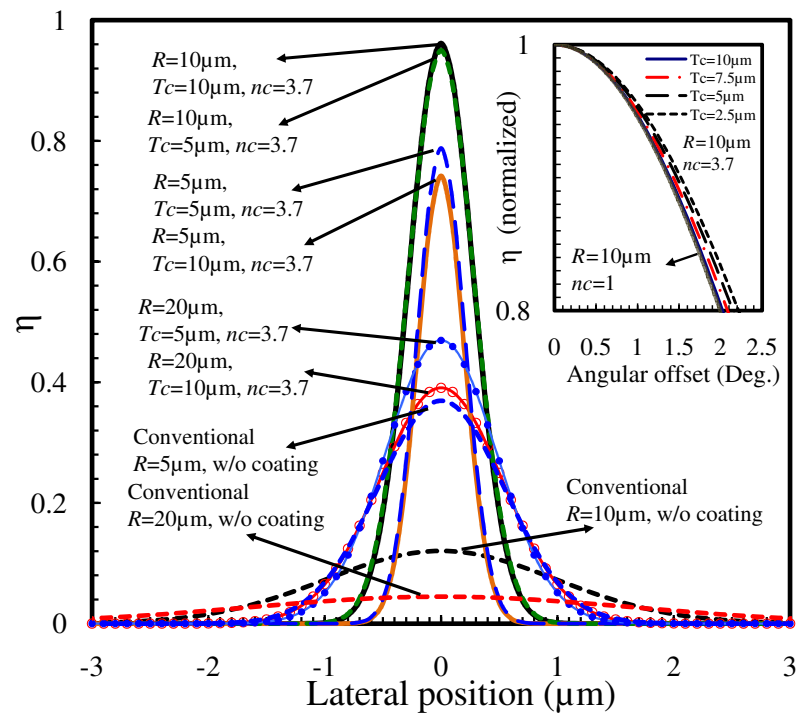

FIG. 5 Variation of coupling efficiency with lateral alignment tolerance for fiber coupling of nanowire waveguide $\left(2 \omega_{0 w}=0.70 \mu \mathrm{m}\right)$ to different lensed single mode fibers with and without coating layers. The inset indicates the normalized coupling efficiency with respect to the angular tolerance.

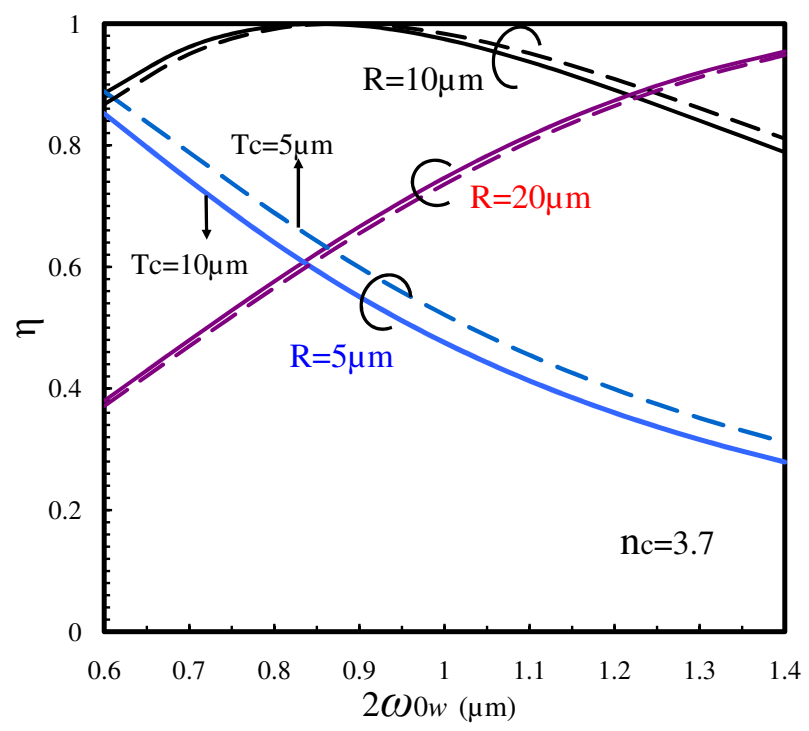

FIG. 6 The calculated coupling efficiency of various waveguide mode sizes coupled to designed higher-index coating lensed single mode fibers coated by a material with an index of 3.7 .

To investigate the alignment tolerance of fiber coupling in the lateral plane, we simulated the coupling efficiency as a function of lateral position for various parameters of lens curvature and coating materials, as shown in Figure 5. Generally, there is a tradeoff when using lensed SMF; the coupling ef- ficiency can be higher, but simultaneously the lateral tolerance is reduced. Compared with conventional lensed SMF, the lateral tolerances of coated ones are tighter in the range of $R=5 \mu \mathrm{m}$ to $20 \mu \mathrm{m}$, while larger lens radius fibers have a slightly larger tolerance. However, the lateral tolerance difference between $R=5 \mu \mathrm{m}$ and $R=20 \mu \mathrm{m}$ is less than $\pm 300 \mathrm{~nm}$ at full width half maximum (FWHM). This shows that the lateral tolerance can be slightly improved by altering parameters such as the radius of curvature of the lensed fibers. In addition, for our investigated coating lensed SMF, coupling efficiency as high as $80 \%$ can be found for angular tolerances in the range of $2^{\circ}$ to $2.3^{\circ}$. The angular tolerance of high index coating SMF is slightly larger than that of conventional lensed SMF and is not sensitive to the coating layer thickness as shown in the inset of Figure 5.

Based on the above numerical analysis, we have designed high-index coating lensed SMF with appropriate parameters of lens curvature and coating index and thickness for nanowire waveguide coupling. We here consider the cases when the coating layer thickness is $5 \mu \mathrm{m}$ or $10 \mu \mathrm{m}$ with an index of 3.7, which is assumed to be $a-\mathrm{Si}: \mathrm{H}$ material, as reported in [6]. The coupling efficiency with various MFD of nanowire waveguides was calculated, as shown in Figure 6. For a high coupling efficiency $(>80 \%)$, the radius of curvature of $10 \mu \mathrm{m}$ can be used for coupling to mode field diameters ranging from $0.6 \mu \mathrm{m}$ to $1.3 \mu \mathrm{m}$ with working distances of $2 \mu \mathrm{m}$ to $5 \mu \mathrm{m}$.

To further validate our numerical modelling analysis based on Gaussian beam and two-lens system approximation, we compared the values of working distance of high-index coating SMF and its coupling efficiency to nanowire waveguide calculated using our method and the 2D-FDTD method reported by Shiraishi et al. [6]. As our method presented in this paper is established for the case of semi-circular lens, the radius of curvature lens is then chosen appropriately as $10.5 \mu \mathrm{m}$ in order to fit (cf. the range of $-4 \mu \mathrm{m}$ to $4 \mu \mathrm{m}$ in core size) the investigated curved lens [6] where the curvature radius and conic constant are $10 \mu \mathrm{m}$ and -2 , respectively. Other parameters such as the MFD of SMF, the fiber core diameter, and the fiber core index are considered to be $10.4 \mu \mathrm{m}, 8.3 \mu \mathrm{m}$, and 1.45 respectively, in both cases. The simulation results show that the working distance is $5.5 \mu \mathrm{m}$ using our method and $5.6 \mu \mathrm{m}$ using 2D-FDTD. When we further consider the coupling between high-index coating SMF and nanowire waveguide with $1.4 \mu \mathrm{m}$ wide MFD, the calculated coupling efficiencies of our case and [6] are estimated to be $91 \%$ and $87 \%$, respectively. Based on above comparison, the simulation deviation is not explicit between two methods within this investigation range; however, the calculated beam profile at focal plane contains 


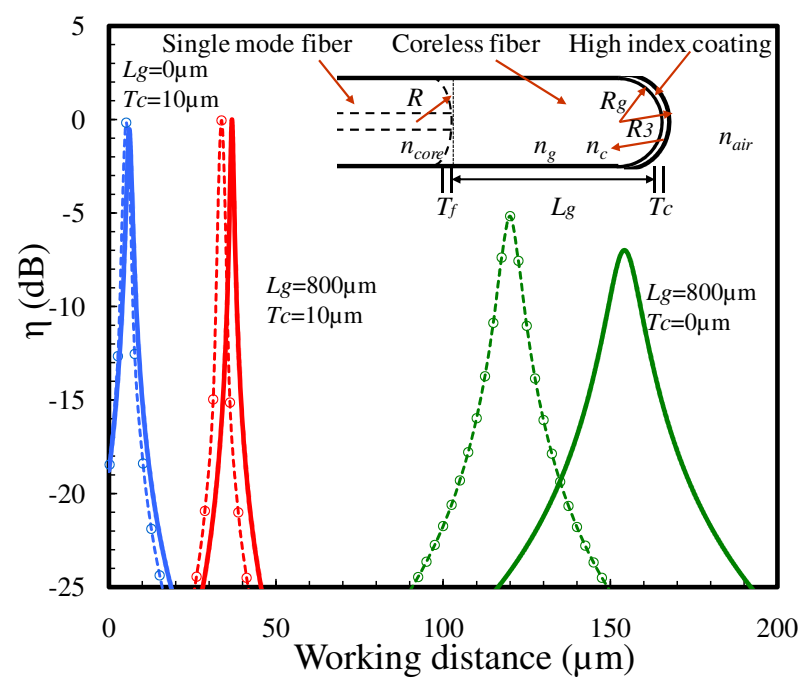

FIC. 7 Variation of coupling loss with working distance for three different lensed single mode fiber designs: high-index coating lensed $\operatorname{SMF}\left(R_{2}=12.5 \mu \mathrm{m}, T_{C}=10 \mu \mathrm{m}\right)$, high-index coating SMF with coreless lensed fiber $\left(R_{g}=60 \mu \mathrm{m}, T_{c}=10 \mu \mathrm{m}\right)$, and non-coating SMF with coreless lensed fiber $\left(R_{g}=60 \mu \mathrm{m}, T_{c}=0 \mu \mathrm{m}\right)$. Dotted lines with circles indicate when phase error $(L)$ is nearly 0.

side lobes with 2D-FDTD method, which may attribute to a slightly lower coupling efficiency value when compared to our calculation.

\section{ENHANCED LENSED FIBER DESIGN}

\subsection{Numerical analysis}

In Section 2, we demonstrated a numerical analysis method which can be used to calculate coupling performances and presented especially the case of fiber coupling from nanowire waveguides to high-index coating lensed SMF. With this scheme, a coupling efficiency higher than $80 \%$ can be obtained when properly designing fiber parameters. However, its short working distance may limit the application on nanophotonics. To further improve the working distance, we propose here another fiber design and evaluate the coupling performances using our developed numerical analysis based on Gaussian beam and two-lens system approximation.

The proposed lensed fiber consists of a SMF, a coreless fiber section, and a high index layer coated on the tip, as shown in the inset of Figure 7. The scheme can be easily fabricated by fusion splicing between the coreless and SMF sections. After fusion splicing, the lensed tip can be formed by electrical discharge on the cleaved end facet of the coreless fiber and then high index silicon would be deposited on the tip. The radius of curvature of the lensed tip by electrical discharge $\left(R_{g}\right)$ is usually in the range of $60 \mu \mathrm{m}$ to $80 \mu \mathrm{m}$. By modifying the equations presented in Section 2, the characteristic ABCD matrix of this high-index coating SMF with a coreless section design can be expressed as

$$
\begin{aligned}
\left(\begin{array}{cc}
A^{\prime} & B^{\prime} \\
C^{\prime} & D^{\prime}
\end{array}\right)= & \left(\begin{array}{cc}
1 & 0 \\
-\frac{1}{f_{\text {coating }}^{\prime}} & 1
\end{array}\right)\left(\begin{array}{cc}
1 & \frac{T_{c}}{n_{c}} \\
0 & 1
\end{array}\right)\left(\begin{array}{cc}
1 & 0 \\
-\frac{1}{f_{g}^{\prime}} & 1
\end{array}\right) \\
& \times\left(\begin{array}{cc}
1 & \frac{L_{g}}{n_{g}} \\
0 & 1
\end{array}\right)\left(\begin{array}{cc}
1 & 0 \\
-\frac{1}{f_{f}^{\prime}} & 1
\end{array}\right)\left(\begin{array}{cc}
1 & \frac{T_{f}}{n_{\text {core }}} \\
0 & 1
\end{array}\right)
\end{aligned}
$$

where $f_{\text {coating }}^{\prime} f_{g}^{\prime}$, and $f_{f}^{\prime}$ are the focal lengths of curved coating layer, curved coreless section, and SMF, respectively, and are expressed as $f_{\text {coating }}^{\prime}=R_{3} /\left(n_{c}-1\right), f_{g}^{\prime}=R_{g} n_{c} /\left(n_{g}-n_{c}\right)$, and $f_{f}^{\prime}=R n_{g} /\left(n_{\text {core }}-n_{g}\right)$. The length of a coreless fiber section is indicated as $L_{g}$. Here, we consider the case of $n_{\text {core }}=$ $1.45, n_{g}=1.5, T_{f}=0$ (as a thin lens), $R=\infty$ (as a cleaved facet), and $R_{3}=R_{f}+T_{c}$. It should be noted that the phase error term can not be ideally small here due to a longer focal length by the large radius of curvature of the lensed tip, which means $r_{f} \neq f$. Therefore, the spot size and wavefront of the lensed fiber are expressed as

$$
\begin{gathered}
\omega_{f}^{\prime}=A^{\prime} \omega_{0 f} \sqrt{1+\left(\frac{\lambda B^{\prime}}{A^{\prime} \pi \omega_{0 f}^{2}}\right)^{2}}, \\
r_{f}^{\prime}=A^{\prime} B^{\prime}\left(\frac{1+\left(A^{\prime} \pi \omega_{0 f}^{2} / \lambda B^{\prime}\right)^{2}}{1+B^{\prime} C^{\prime}\left\{1+\left(A^{\prime} \pi \omega_{0 f}^{2} / \lambda B^{\prime}\right)^{2}\right\}}\right) .
\end{gathered}
$$

When the light exits from the SMF core, it enters first the coreless fiber section and its beam diameter is expanded. As the beam further propagates, part of the beam hits the air-glass interface and reflects back into the coreless fiber; therefore, multimode interference patterns can be generated. To minimize the multimode interference, we choose the length of coreless fiber section as short as $900 \mu \mathrm{m}$ for the coreless fiber diameter of $125 \mu \mathrm{m}$ in this calculation.

\subsection{Simulation results and discussion}

In Figure 7, we show the comparison of coupling efficiency with respect to working distance for three different lensed fiber designs including our proposed high index coating lensed SMF with a coreless fiber tip, the lensed SMF with high index coating [6], and the non-coating lensed SMF with a coreless tip [13]. For the coated fibers, we considered the case with a coating thickness of $10 \mu \mathrm{m}$ and a coating layer index of 3.7, which is assumed to be a-Si:H. Generally, the high index coating lensed SMFs show good coupling efficiency characteristic, as high as $80 \%$, however the working distance is less than $5 \mu \mathrm{m}$. In the case of a non-coating lensed SMF with a coreless tip, the working distance can increase to $\sim 160 \mu \mathrm{m}$ with a theoretical coupling loss value of $\sim 7 \mathrm{~dB}$. Usually, a long working distance is preferred in order to avoid damaging the nanowire facet during assembling or alignment. Nevertheless, the mode size of the nanowire waveguide at $\sim 160 \mu \mathrm{m}$ working distance is larger than the fiber diameter (125 $\mu \mathrm{m}$ wide) due to diffraction when the beam arrives at the fiber tip; the coupling loss thus increases because of over-expanded mode size. If we now consider only the coupling limitations coming from the beam divergence angle, the working distance must be shorter than $45 \mu \mathrm{m}$ when $2 \omega_{0 w(x, y)}=0.7 \mu \mathrm{m}$. From the simulated results, our design of high index coating SMF with coreless fiber section actually shows a working distance of $36.8 \mu \mathrm{m}$ with a coupling loss less than $0.5 \mathrm{~dB}$. In Figure 7, we present as well the coupling efficiency differences when calculations are done with considering ideal ( $L$ is nearly zero) and non-ideal phase error terms. For a longer working distance, the phase error term may not be small and it would lead to incorrect coupling efficiency calculation results. In short working distance, the coupling efficiency is calculated to be slightly higher, however, in the 


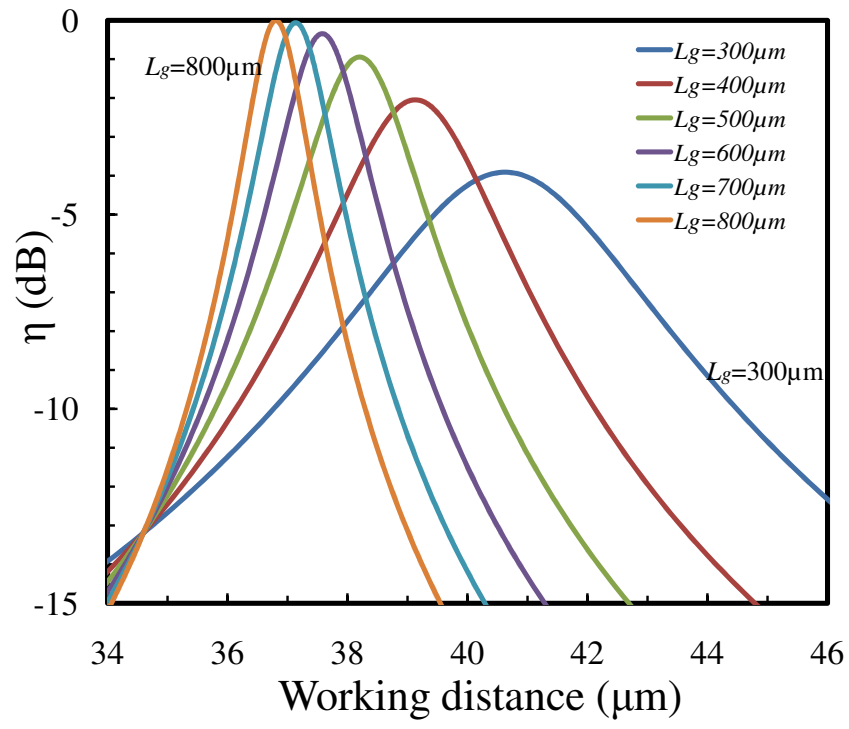

FIC. 8 Variation of coupling loss with working distance for various lengths of coreless fiber sections for coupling from nanowire with $0.7 \mu \mathrm{m}$ wide mode size diameter to high-index coating lensed SMF with coreless lensed fiber $\left(R_{g}=60 \mu \mathrm{m}\right)$.

case of non-coating lensed SMF with a coreless tip, there is $2 \mathrm{~dB}$ difference when applying two different phase error terms.

Figure 8 shows the coupling loss as a function of working distance while varying the length of the coreless fiber section $\left(L_{g}\right)$. We can observe that the longitudinal tolerance is inversely proportional to the length of the coreless fiber section. For example, the $1 \mathrm{~dB}$ longitudinal tolerance is $2 \mu \mathrm{m}$ when $L_{g}=300 \mu \mathrm{m}$ and it decreases to $\sim 0.7 \mu \mathrm{m}$ when $L_{g}=800 \mu \mathrm{m}$. This means that there is a tradeoff between the coupling loss and longitudinal tolerance when choosing the length of coreless fiber section. Our optimized design predicts a coupling loss of nearly $0 \mathrm{~dB}$ with a working distance of $36.8 \mu \mathrm{m}$ for $800 \mu \mathrm{m}$ long coreless fiber section. This proposed coreless lensed fiber with hight index coating can compensate the drawbacks of short working distance and low coupling efficiency of two other types of lensed SMF discussed above.

\section{CONCLUSION}

In this paper, we present a nanowire waveguide coupling to high-index coating lensed single mode fibers using an alternative numerical analysis of mode overlap integral method based on Gaussian beam and two-lens approximation. For practical use with coupling efficiency higher than $80 \%$, the lensed SMF with radius of curvature of $10 \mu \mathrm{m}$ and coated with $10 \mu \mathrm{m}$ thick high-index material can be used to couple waveguides with $0.6 \mu \mathrm{m}$ to $1.3 \mu \mathrm{m}$ wide mode size diameters. Furthermore, by inserting an additional coreless fiber section into the high-index coating lensed SMF, the working distance can be further improved. For a Si-nanowire waveguide with $0.7 \mu \mathrm{m}$ wide mode size diameter, our numerical simulations show that the coupling loss can be as low as $0.5 \mathrm{~dB}$ with a long working distance of $36.8 \mu \mathrm{m}$ when using the optimized structure in which the coreless fiber section has a radius of curvature of $60 \mu \mathrm{m}$, a length of $800 \mu \mathrm{m}$, and coated with a
$10 \mu \mathrm{m}$ thick high index layer with index of 3.7. We believe that this numerical analysis would serve as a guide for designing lensed fibers and our enhanced design structure can be a viable solution for fiber coupling with Si-based nanowire waveguides.

\section{ACKNOWLEDGEMENTS}

This work was supported by the Science Foundation Ireland under Grant 07/SRC/I1173.

\section{References}

[1] L. Zimmermann, T. Tekin, H. Schroeder, P. Dumon, and W. Bogaerts, "How to bring nanophotonics to application-silicon photonics packaging" IEEE LEOS News. 22, 4-14 (2008).

[2] G. Roelkens, D. V. Thourhout, and R. Baets, "High efficiency grating coupler between silicon-on-insulator waveguides and perfectly vertical optical fibers" Opt. Lett. 32, 1495-1497 (2007).

[3] R. Halir, P. Cheben, S. Janz, D.-X. Xu, I. Molina-Fernández, and J. G. Wangüemert-Pérez, "Waveguide grating coupler with subwavelength microstructures" Opt. Lett. 34, 1408-1410 (2009).

[4] K. Shiraishi, H. Yoda, A. Ohshima, H. Ikedo, and C. S. Tsai, "A silicon-based spot-size converter between single-mode fibers and Si-wire waveguides using cascaded tapers" Appl. Phys. Lett. 91, 141120-1-3 (2007).

[5] C. Pang, F. Gesuele, A. Bruyant, S. Blaize, C. Lerondel, and P. Royer, "Enhanced light coupling in sub-wavelength single-mode silicon on insulator waveguides" Opt. Express 17, 6939-6945 (2009).

[6] K. Shiraishi, N. Kawasaki, H. Yoda, K. Watanabe, M. Umetsu, T. Hitomi, and K. Muro, "High-index-layer coating on a lensed fiber endface for enhanced focusing power" J. Lightwave Technol. 27, 864870 (2009).

[7] H. Kogelnik, and T. Li, “Laser beams and resonators" Appl. Opt. 5, 1550-1567 (1966).

[8] J. H. Song, H. N. J. Fernando, B. Roycroft, B. Corbett, and F. H. Peters, "Practical design of lensed fibers for semiconductor laser packaging using laser welding technique" J. Lightwave Technol. 27, 1533-1539 (2009).

[9] C. A. Edwards, H. M. Presby, and C. Dragone, “Ideal microlenses for laser to fiber coupling" J. Lightwave Technol. 11, 252-257 (1993).

[10] K. S. Lee, and F. S. Barnes, "Microlenses on the end of singlemode optical fibers for laser applications" Appl. Opt. 24, 3134-3139 (1985).

[11] J. H. Song, P. O'Brien, and F. H. Peters, "Optimal laser welding assembling sequences for butterfly laser module packages" 0 pt. Express 17, 16406-16414 (2009).

[12] J. H. Song, K.-Y. Kim, J. Cho, D. Han, J. Lee, Y. S. Lee, S. Jung, Y. Oh, D.-H. Jang, and K. S. Lee, "Thin film filter-embedded triplexingfilters based on directional couplers for FTTH networks" IEEE Photonic. Tech. Lett. 17, 1668-1670 (2005).

[13] K. Shiraishi, N. Oyama, K. Matsumura, I. Ohishi, and S. Suga, “A fiber lens with a long working distance for integrated coupling between laser diode and single mode fiber" J. Lightwave Technol. 13, 1736-1744 (1995). 